\title{
ON QUASICONFORMAL HARMONIC MAPS
}

\author{
Luen-FaI TAM AND TOM Y.-H. WAN
}

\begin{abstract}
It was proved by the authors that given a quasiconformal harmonic diffeomorphism $F$ on $\mathbb{H}^{2}$, there is a neighborhood $\mathcal{N}$ of the class $\bar{F}$ represented by $F$ in the universal Teichmüller space such that if $\bar{H} \in \mathcal{N}$, then the boundary map of $H$ can be extended to a quasiconformal harmonic diffeomorphism on $\mathbb{H}^{2}$, i.e. the class $\bar{H}$ can be represented by a quasiconformal harmonic diffeomorphism. More precisely, it was proved that if $F$ is a quasiconformal harmonic diffeomorphism on $\mathbb{H}^{2}$, and if $G$ is a quasiconformal map on $\mathbb{H}^{2}$ such that the dilatation of $G$ is small enough, then there exists quasiconformal harmonic diffeormophisms with the same boundary data with $F \circ G$ and $G \circ F$. The purposes of this paper is to study the higher dimensional generalization to this result and related problems.
\end{abstract}

\section{Introduction.}

In $[\mathbf{W}]$, the second author showed that quasiconformal harmonic diffeomorphisms on $\mathbb{H}^{2}$ can be parametrized by bounded holomorphic quadratic differentials on $\mathbb{H}^{2}$. This gives a map from the space of bounded holomorphic quadratic differentials on $\mathbb{H}^{2}$ into the universal Teichmüller space. It was conjectured by Schoen $[\mathbf{S}]$ that the map is a bijection. The conjecture of Schoen can be rephrased as follow: Every quasi-symmetric map on $\mathbb{S}^{1}$ can be extended uniquely to a quasiconformal harmonic diffeomorphism on the two dimensional real hyperbolic space $\mathbb{H}^{2}$. Here a quasi-symmetric map on $\mathbb{S}^{1}$ can be defined as the boundary map on the unit disk in $\mathbb{C}$ of a quasiconformal homeomorphism, see $[\mathbf{B}-\mathbf{A}]$. The uniqueness part of the conjecture was proved by Li and the first author [L-T 3]. As for existence, the problem is still open. However, there are many partial results. For example, it was proved by Li and the first author in [L-T 3] that existence part of Schoen's conjecture is true for $C^{1}$ diffeomorphisms on $\mathbb{S}^{1}$. For more partial results, see $[\mathbf{C}-\mathbf{T}, \mathbf{L}-\mathbf{W g}, \mathbf{W g}]$ for example.

There is one important fact for harmonic map in two dimensions which is very likely not be true in higher dimensions. Namely, if a harmonic map on $\mathbb{H}^{2}$ is such that the boundary map is a homeomorphism on $\mathbb{S}^{1}$, then the harmonic map is itself a diffeomorphism. In a recent paper, Li and Wang 
[L-Wg] study a larger class of harmonic maps which seems more natural in the higher dimensions. Before we go further, let us introducing some definitions.

Definition 0.1. Let $u$ be a map from a metric space $M$ to another metric space $N$ :

- $\quad u$ is said to be a rough-isometry if there exists positive constants $a$ and $b$ such that for all $x, y \in M$

$$
a^{-1} d_{M}(x, y)-b \leq d_{N}(u(x), u(y)) \leq a d_{M}(x, y)+b .
$$

- $u$ is said to be a pseudo-isometry if there exists positive constants a and $b$ such that for all $x, y \in M$

$$
a^{-1} d_{M}(x, y)-b \leq d_{N}(u(x), u(y)) \leq a d_{M}(x, y) .
$$

- $u$ is said to be a quasi-isometry if $u$ is a homeomorphism and there exists positive constant $a>0$ such that for all $x, y \in M$

$$
a^{-1} d_{M}(x, y) \leq d_{N}(u(x), u(y)) \leq a d_{M}(x, y) .
$$

It is obvious that a quasi-isometry is a pseudo-isometry and a pseudoisometry is a rough isometry. Pseudo-isometries have been used by Mostow $[\mathrm{M}]$ in the proof of strong rigidity of locally symmetric spaces. Note that while a pseudo-isometry is Lipschitz continuous, a rough-isometry may not even be continuous. On the other hand, if $u$ is a rough-isometric harmonic map on $\mathbb{H}^{n}$, satisfying (0.1), then $u\left(B_{x}(r)\right) \subset B_{u(x)}(a r+b)$. By the estimate of energy density for harmonic maps by Cheng [C], $u$ has bounded energy density and hence $u$ is a pseudo-isometry. By $[\mathbf{M}], u$ is then surjective. By the results of $[\mathbf{M}]$ again, $u$ can be extended to a quasiconformal map on $\mathbb{S}^{n-1}$ which is identified with the geometric boundary of $\mathbb{H}^{n}$. Roughisometric harmonic maps have been studied in a recent paper $[\mathbf{L}-\mathbf{W g}]$ of $\mathrm{Li}$ and Wang. They generalize the conjecture of Schoen to the following: Every quasiconformal map on the geometric boundary of a rank-1 symmetric space of noncompact type can be extended uniquely to a harmonic rough-isometry. In $[\mathbf{L}-\mathbf{W g}]$, the uniqueness part of the conjecture is proved.

In this paper, we will discuss quasiconformal harmonic maps on $\mathbb{H}^{n}$ for $n \geq 3$. A quasiconfomal map on $\mathbb{H}^{n}$ is a rough-isometry, see $[\mathbf{P}]$. Hence our results will be related to Li-Wang conjecture on hyperbolic space of dimension greater than two. We will prove that given a quasiconformal harmonic diffeomorphism $F$ on $\mathbb{H}^{n}$, there is a $K_{0}>1$ such that if $G$ is a $K_{0}$-quasiconformal map on $\mathbb{S}^{n-1}$, then there is a pseudo-isometric harmonic map on $\mathbb{H}^{n}$ with the same boundary data as $G \circ F$. If, in addition, $F$ is a 
quasi-isometry, the same conclusion is true for boundary data $F \circ G$. These results can be considered as generalizations of the results in $[\mathbf{T}-\mathbf{W}]$ for $\mathbb{H}^{2}$ to higher dimensions. As mentioned before, in [L-T 3], it was proved that any $C^{1}$ diffeomorphism on $\mathbb{S}^{n-1}$ can be extended to a harmonic map $F$ on $\mathbb{H}^{n}$ which is $C^{1}$ up to the geometric boundary. Such a map may not be a diffeomorphism, and in particular, may not be quasiconformal. However, we will prove that such a harmonic map is a pseudo-isometry and in fact it is a quasi-isometry near infinity (see Definition 3.1). We will also prove that one can find pseudo-isometric harmonic maps with the same boundary data as $F \circ G$ and $G \circ F$ respectively, for any $K_{0}$-quasiconformal map $G$ on $\mathbb{S}^{n-1}$, provided $K_{0}>1$ is small enough. Note that in this case, $K_{0}$ does not depend on $F$, while in the previous case of quasiconformal harmonic diffeomorphism, the number $K_{0}$ depends on the map $F$.

One can get more information on those harmonic maps. In $[\mathbf{W}]$, the second author proved that a harmonic diffeomorphism on $\mathbb{H}^{2}$ is quasiconformal if and only if it has uniformly bounded energy density. In this case, the harmonic map is actually a quasi-isometry. In high dimensions, the situation is more complicated. However, we will prove that the harmonic maps constructed above are actually quasi-isometries, provided $K_{0}>1$ is even smaller, and the given map $F$ is a quasi-isometry to begin with. In the case of harmonic maps which are $C^{1}$ up to the boundary, the 'nearby' harmonic maps are also quasi-isometries near infinity.

In particular, if we choose the given map to be an isometry, we can conclude that if $G$ is a $K_{0}$-quasiconformal with $K_{0}>1$ small enough, then $G$ can be extended to a quasi-isometric harmonic diffeomorphism on $\mathbb{H}^{n}$. By the uniqueness theorem in $[\mathbf{L}-\mathbf{W g}]$, we see that every quasiconformal harmonic map on $\mathbb{H}^{n}$ with small quasiconformal constant must be a quasi-isometry. This is a partial generalization of the result in $\mathbb{H}^{2}$ by the second author $[\mathbf{W}]$. In fact, using other method, we will give an explicit estimate for the quasiconformal constant $K_{0}$ so that every $K_{0}$-quasiconformal harmonic map on $\mathbb{H}^{n}$ is a quasi-isometry. We should made precise the meaning of the quasiconformal constant $K_{0}$ here since there are several equivalent definitions for quasiconformal mappings with slightly different meaning of the quasiconformal constant (i.e. the maximal dilatation). Note that all other results in this paper do not depends on the specific choice of the definition of $K_{0}$. We choose the following:

Definition 0.2. A smooth positively oriented homeomorphism $F$ from the unit ball $\mathbf{B}^{n}$ in $\mathbb{R}^{n}$ onto itself is said to be a $K$-quasiconformal map, if for all $x \in \mathbf{B}^{n},\|d F\|^{n}(x) \leq K J(x)$ and $J(x) \leq K\{\ell(d F)(x)\}^{n}$, where

$$
\|d F\|(x)=\max _{|\vec{v}|=1}|d F(x)(\vec{v})|
$$


and

$$
\ell(d F)(x)=\min _{|\vec{v}|=1}|d F(x)(\vec{v})|
$$

The paper is organized as follows: In $\S 1$, we will study some basic properties of the Douady-Earle extensions $[\mathbf{D}-\mathbf{E}]$ on the unit ball in $\mathbb{R}^{n}$ of quasiconformal map on $\mathbb{S}^{n-1}$ which will be useful in later sections. In $\S 2$, and $\S 3$, we will construct pseudo-isometric harmonic maps with boundary data which are near some given harmonic maps. Finally, in $\S 4$, we will discuss the properties of the harmonic maps constructed in the previous two sections and study some properties of general quasiconformal harmonic maps.

Added in proof: The first part of Theorem 2.6 for quasi-isometric harmonic maps is also obtained by Hardt and Wolf $[\mathbf{H}-\mathbf{W}]$. The case of isometries is obtained by D. Yang $[\mathbf{Y}]$ independently too.

\section{Estimates on Douady-Earle extension.}

In this section, we will give estimates on the derivatives of Douady-Earle extension $[\mathbf{D}-\mathbf{E}]$ of quasiconformal self maps on $\mathbb{S}^{n-1}$. We first fix our notation. Let $\theta_{i}, 1 \leq i \leq m$, be a local orthonormal coframe of $\mathbb{H}^{m}$ and $\theta_{i j}, 1 \leq i, j \leq m$, be the connection forms. Similarly, let $\omega_{\alpha}$ and $\omega_{\alpha \beta}$, $1 \leq \alpha, \beta \leq n$, be local orthonormal coframe and the corresponding connection forms of $\mathbb{H}^{n}$. For a smooth map $F: \mathbb{H}^{m} \rightarrow \mathbb{H}^{n}$, one define the energy density of $F$ by

$$
e(F)=\sum_{i, \alpha}\left(f_{i}^{\alpha}\right)^{2}
$$

where $f_{i}^{\alpha}$ is given by $F^{*}\left(\omega_{\alpha}\right)=\sum_{i} f_{i}^{\alpha} \theta_{i}$. The Hessian of the map $F$, denoted by $f_{i j}^{\alpha}$, is defined by

$$
\sum_{j} f_{i j}^{\alpha} \theta_{j}=d f_{i}^{\alpha}-\sum_{j} f_{j}^{\alpha} \theta_{i j}+\sum_{\beta} f_{i}^{\beta} F^{*}\left(\omega_{\beta \alpha}\right),
$$

and we write $\left|\nabla^{2} F\right|^{2}=\sum_{i, j, \alpha}\left(f_{i j}^{\alpha}\right)^{2}$ for its norm. Finally, the components of the tension field of $F$ is defined by $\tau^{\alpha}(F)=\sum_{i} f_{i i}^{\alpha}, 1 \leq \alpha \leq n$, and its norm by $|\tau(F)|=\left(\sum_{\alpha}\left(\tau^{\alpha}\right)^{2}\right)^{1 / 2}$.

The Douady-Earle extension [D-E] is a conformally natural extension for homeomorphisms from unit sphere onto itself. The construction is as follows. First, they define the barycenter $B(\mu)$ of a probability measure $\mu$ on $\mathbb{S}^{n-1}$ with no atoms as the unique zero of the hyperbolic gradient of

$$
h_{\mu}(x)=\frac{1}{2} \int_{\mathbb{S}^{n-1}} \log \frac{1-|x|^{2}}{|x-u|^{2}} \mathrm{~d} \mu(u), \quad x \in \mathbf{B}^{n},
$$


where $\mathbf{B}^{n}$ is the open unit ball in $\mathbb{R}^{n}$. Then they define the extension $\mathcal{E}(\varphi)$ of $\varphi: \mathbb{S}^{n-1} \rightarrow \mathbb{S}^{n-1}$ to $\bar{B}^{n}$ by

$$
\mathcal{E}(\varphi)(x)=B\left(\varphi_{*}\left(\eta_{x}\right)\right), \quad x \in \mathbf{B}^{n} .
$$

In the formula, $\varphi_{*}\left(\eta_{x}\right)(E)=\eta_{x}\left(\varphi^{-1} E\right)$, for all Borel subset $E \subset \mathbb{S}^{n-1}$, and $\eta_{x}$ is the hyperbolic harmonic measure on $\mathbb{S}^{n-1}$ given by

$$
\eta_{x}(E)=\frac{1}{\omega_{n-1}} \int_{E}\left(\frac{1-|x|^{2}}{|x-u|^{2}}\right)^{n-1} \mathrm{~d} \omega(u),
$$

where $\mathrm{d} \omega$ and $\omega_{n-1}$ are the standard measure and volume on $\mathbb{S}^{n-1}$.

The main results concerning the high dimensional $(\geq 3)$ case are collected in the following:

Theorem 1.1 (Douady-Earle $[\mathrm{D}-\mathrm{E}])$. Let $\mathcal{H}\left(\mathbb{S}^{n-1}\right)(n \geq 3)$ be the set of homeomorphisms from $\mathbb{S}^{n-1}$ onto itself. Then $\mathcal{E}$ is a mapping from $\mathcal{H}\left(\mathbb{S}^{n-1}\right)$ into $\mathcal{C}^{\infty}\left(\mathbf{B}^{n}, \mathbb{R}^{n}\right) \cap \mathcal{C}\left(\bar{B}^{n}, \mathbb{R}^{n}\right)$ satisfying:

(i) For any $\varphi \in \mathcal{H}\left(\mathbb{S}^{n-1}\right),\left.\mathcal{E}(\varphi)\right|_{\mathbb{S}^{n-1}}=\varphi$.

(ii) Let $\operatorname{Isom}\left(\mathbb{H}^{n}\right)$ be the isometry group of $\mathbf{B}^{n}$ with respect to the Poincaré metric, then $\mathcal{E}$ is equivariant under $\operatorname{Isom}\left(\mathbb{H}^{n}\right)$, i.e. for all $\gamma, \sigma \in$ $\operatorname{Isom}\left(\mathbb{H}^{n}\right)$,

$$
\mathcal{E}(\gamma \circ \varphi \circ \sigma)=\gamma \circ \mathcal{E}(\varphi) \circ \sigma
$$

(iii) The mapping $\mathcal{E}: \mathcal{H}\left(\mathbb{S}^{n-1}\right) \rightarrow \mathcal{C}^{\infty}\left(\mathbf{B}^{n}, \mathbb{R}^{n}\right) \cap \mathcal{C}\left(\bar{B}^{n}, \mathbb{R}^{n}\right)$ is continuous, where $\mathcal{H}\left(\mathbb{S}^{n-1}\right)$ and $\mathcal{C}\left(\bar{B}^{n}, \mathbb{R}^{n}\right)$ have the uniform topology, $\mathcal{C}^{\infty}\left(\mathbf{B}^{n}, \mathbb{R}^{n}\right)$ has the $\mathcal{C}^{\infty}$ topology, and $\mathcal{C}^{\infty}\left(\mathbf{B}^{n}, \mathbb{R}^{n}\right) \cap \mathcal{C}\left(\bar{B}^{n}, \mathbb{R}^{n}\right)$ has the induced topology regarded as the diagonal of the product space with product topology.

Proof. Please refer to the final section of [D-E] for the proof.

In order to apply the Douady-Earle extension to the existence of harmonic maps, we need estimates on the gradients and the Hessian of $\mathcal{E}(\varphi)$ when $\varphi$ closes to conformal, namely, $\varphi$ is a $K$-quasiconformal mapping with $K$ closes to 1 . More precisely, we have:

Proposition 1.2. Let $\left(\mathcal{E}(\varphi)_{j}^{i}\right)$ and $\left|\nabla^{2} \mathcal{E}(\varphi)\right|$ be the Jacobian matrix and the norm of the Hessian of $\mathcal{E}(\varphi)$ with respect to orthonormal frames of the Poincaré metric respectively. Then for any $\epsilon>0$, there exists $K>1$ depending only on $\epsilon$ and $n$ such that if $\varphi$ is $K$-quasiconformal, then

(i) (Tukia) $\mathcal{E}(\varphi): \bar{B}^{n} \rightarrow \bar{B}^{n}$ is a quasiconformal homeomorphism and

$$
\begin{aligned}
& (1+\epsilon)^{-1} d(x, y) \leq d(\mathcal{E}(\varphi)(x), \mathcal{E}(\varphi)(y)) \leq(1+\epsilon) d(x, y) \\
& \text { for all } x, y \in \mathbf{B}^{n},
\end{aligned}
$$

where $d$ is the Poincaré distance in $\mathbf{B}^{n}$. 
(ii)

$$
\sqrt{\sum_{j, k}\left(\sum_{i} \mathcal{E}(\varphi)_{i}^{j} \mathcal{E}(\varphi)_{i}^{k}-\delta_{j k}\right)^{2}}<\epsilon
$$

and

(iii)

$$
\left|\nabla^{2} \mathcal{E}(\varphi)\right|<\epsilon
$$

Proof. (i) is proved in [D-E] by Tukia. Actually the proof works for (i)-(iii). To be precise, let

$$
\begin{aligned}
& \alpha(\varphi)(x)=\inf _{u \in \mathbb{S}^{n-1}} \frac{\left(1-|x|^{2}\right)|\mathrm{d} \mathcal{E}(\varphi)(x) u|}{1-|\mathcal{E}(\varphi)(x)|^{2}}, \\
& \beta(\varphi)(x)=\sup _{u \in \mathbb{S}^{n-1}} \frac{\left(1-|x|^{2}\right)|\mathrm{d} \mathcal{E}(\varphi)(x) u|}{1-|\mathcal{E}(\varphi)(x)|^{2}},
\end{aligned}
$$

and

$$
\Lambda(\varphi)=\sqrt{\sum_{j, k}\left(\sum_{i} \mathcal{E}(\varphi)_{i}^{j} \mathcal{E}(\varphi)_{i}^{k}-\delta_{j k}\right)^{2}} .
$$

We note that (i) follows from the statement that for any $\epsilon>0$, there exists $K>1$ depending only on $\epsilon$ and $n$ such that

$$
(1+\epsilon)^{-1}<\alpha(\varphi) \leq \beta(\varphi)<1+\epsilon
$$

provided that $\varphi$ is $K$-quasiconformal. Hence all statements (i)-(iii) can be regarded as estimates of the derivatives of $\mathcal{E}(\varphi)$.

Then we observe that for all $\gamma$ and $\sigma \in \operatorname{Isom}\left(\mathbb{H}^{n}\right)$,

$$
\begin{gathered}
\alpha(\gamma \circ \varphi \circ \sigma)=\alpha(\varphi) \circ \sigma, \quad \beta(\gamma \circ \varphi \circ \sigma)=\beta(\varphi) \circ \sigma, \\
\Lambda(\gamma \circ \varphi \circ \sigma)=\Lambda(\varphi) \circ \sigma,
\end{gathered}
$$

and

$$
\left|\nabla^{2} \mathcal{E}(\gamma \circ \varphi \circ \sigma)\right|=\left|\nabla^{2} \mathcal{E}(\varphi)\right| \circ \sigma
$$

Therefore we only need to estimate these quantities at one point (for all normalized boundary data) to obtain the uniform bounds. More precisely, we 
only need to show that there exists $K>1$ such that, if $\varphi$ is $K$-quasiconformal and fixes the points $e_{1},-e_{1}$, and $e_{n}$, then

$$
\begin{gathered}
(1+\epsilon)^{-1}<\alpha(\varphi)(0) \leq \beta(\varphi)(0)<1+\epsilon, \\
\Lambda(\varphi)(0)<\epsilon
\end{gathered}
$$

and

$$
\left|\nabla^{2} \mathcal{E}(\varphi)\right|(0)<\epsilon
$$

here $\left\{e_{1}, \ldots, e_{n}\right\}$ is the standard ordered basis of $\mathbb{R}^{n}$.

Suppose on the contrary that it is not true, then there exists a sequence of $\varphi_{j}$ of $K_{j}$-quasiconformal mappings from $\mathbb{S}^{n-1}$ onto itself fixing $e_{1},-e_{1}$, and $e_{n}$ with $K_{n} \rightarrow 1$ such that

$$
\begin{aligned}
& \alpha\left(\varphi_{j}\right)(0) \leq(1+\epsilon)^{-1}<1 \\
& \beta\left(\varphi_{j}\right)(0) \geq 1+\epsilon>1 \\
& \Lambda\left(\varphi_{j}\right)(0) \geq \epsilon>0
\end{aligned}
$$

or

$$
\left|\nabla^{2} \mathcal{E}\left(\varphi_{j}\right)\right|(0) \geq \epsilon>0
$$

Then by compactness, we have a subsequence $\varphi_{j_{k}}$ converges to an element $g \in \operatorname{Isom}\left(\mathbb{H}^{n}\right)$ in $\mathcal{H}\left(\mathbb{S}^{n-1}\right)$. By Theorem 1.1 and that $g$ is an isometry, we have

$$
\begin{array}{r}
1>(1+\epsilon)^{-1} \geq \lim _{k \rightarrow \infty} \alpha\left(\varphi_{j_{k}}\right)(0)=\alpha(g)(0)=1, \\
1<1+\epsilon \leq \lim _{k \rightarrow \infty} \beta\left(\varphi_{j_{k}}\right)(0)=\beta(g)(0)=1, \\
0<\epsilon \leq \lim _{k \rightarrow \infty} \Lambda\left(\varphi_{j_{k}}\right)(0)=\Lambda(g)(0)=0
\end{array}
$$

or

$$
0<\epsilon \leq \lim _{k \rightarrow \infty}\left|\nabla^{2} \mathcal{E}\left(\varphi_{j_{k}}\right)\right|(0)=\left|\nabla^{2} \mathcal{E}(g)\right|(0)=0,
$$

which are contradictions.

Remark 1.3. All the above results hold in two dimensions. In fact, if $n=2$, we have a stronger result that the extension $\mathcal{E}(\varphi)$ is quasi-isometric with respect to the Poincaré metric, and hence quasiconformal, provided $\varphi$ is quasi-symmetric. No smallness assumption on the dilatation of the boundary maps is needed. 


\section{Construction of pseudo-isometric harmonic maps (I).}

In this and the next section, we are going to use the results in $\S 1$ to construct pseudo-isometric harmonic maps with certain given boundary data at the geometric boundary of the hyperbolic space. We will generalize the results in $[\mathbf{T}-\mathbf{W}]$ for $\mathbb{H}^{2}$ to higher dimensional hyperbolic spaces. Our main purpose is to prove the existence of harmonic extensions for quasiconformal maps on the geometric boundary of $\mathbb{H}^{m}$ which are near (in certain sense) to those already known to have harmonic extensions. In this section, we will consider those boundary maps which are near to a boundary maps having diffeomorphic quasiconformal harmonic extension. In the next section, we will consider other kind of boundary maps which are near to a $C^{1}$ boundary map with nonvanishing energy density. First, we will give some crucial estimates for the composition of maps. Since composition of maps is not commutative, we have to consider two different cases. In fact, the results are slightly different from each other.

Lemma 2.1. Let $F$ be a harmonic map from $\mathbb{H}^{m}$ to $\mathbb{H}^{n}$. Then for any point $x \in \mathbb{H}^{m}$ and $r>0$, there is a constant $C$ depending only on $m, n, r$ and $\sup _{B_{x}(r)} e(F)$ such that $\left|\nabla^{2} F\right|^{2}(x) \leq C$.

Proof. Since it is easy to see that $F\left(B_{x}(r)\right) \subset B_{F(x)}(\rho)$ with $\rho=$ $r \sup _{B_{x}(r)} \sqrt{e(F)}$ and $F$ is harmonic, the lemma follows from the harmonic map equations and the interior Schauder estimates.

Lemma 2.2. Let $F$ be a harmonic map from $\mathbb{H}^{m}$ to $\mathbb{H}^{n}$ and $G$ be a quasiisometry on $\mathbb{H}^{m}$ such that, for some $\epsilon>0$,

$$
|\tau(G)|<\epsilon \quad \text { and } \sqrt{\sum_{j, k}\left(\sum_{i} g_{i}^{j} g_{i}^{k}-\delta_{j k}\right)^{2}}<\epsilon,
$$

where $g_{i}^{j}$ are components of $d G$ with respect to some local orthonormal coframes. Then, for any $x \in \mathbb{H}^{m}$ and $r>0$, there is a constant $C_{1}$ depending only on $m, n, r$ and $\sup _{B_{x}(r)} e(F)$ such that

$$
|\tau(F \circ G)(x)| \leq C_{1} \epsilon .
$$

In particular, if $F$ has bounded energy density, then

$$
|\tau(F \circ G)| \leq C_{1} \epsilon
$$

with $C_{1}$ now depends only on $m, n$ and upper bound of the energy density of F.

Proof. Let $f_{i}^{\alpha}$ be the components of $d F$ with respect to some local orthonormal coframe on the target and the same local orthonormal coframe on domain which gives $g_{i}^{j}$. And also let $\tau^{\alpha}$ be the corresponding components of 
the tension field of $F \circ G$. Then, by Lemma 2.1 and the assumption that $F$ is harmonic,

$$
\begin{aligned}
\left|\tau^{\alpha}\right| & =\left|\sum_{i, j} f_{j}^{\alpha} g_{i i}^{j}+\sum_{i, j, k} f_{j k}^{\alpha} g_{i}^{j} g_{i}^{k}\right| \\
& =\left|\sum_{j} f_{j}^{\alpha} \tau^{j}(G)+\sum_{j, k} f_{j k}^{\alpha}\left(\sum_{i} g_{i}^{j} g_{i}^{k}-\delta_{j k}\right)\right| \\
& \leq C_{1} \epsilon,
\end{aligned}
$$

for some constant $C_{1}$ depends only on $m, n$ and the local upper bound of the energy density of $F$.

Lemma 2.3. Let $F$ be as in Lemma 2.2 and $G$ be a quasi-isometry from $\mathbb{H}^{n}$ to $\mathbb{H}^{n}$ such that

$$
\left|\nabla^{2} G\right|<\epsilon
$$

for some $\epsilon>0$. Then

$$
|\tau(G \circ F)|(x) \leq \epsilon \cdot e(F)(x), \quad \forall x \in \mathbb{H}^{m} .
$$

Proof. Let $f_{i}^{\alpha}, g_{\beta}^{\alpha}$, and $g_{\beta \gamma}^{\alpha}$ be the components of $d F, d G$ and the components of the Hessian of $G$ with respect to some local orthonormal coframes respectively. Then, since $F$ is harmonic,

$$
\begin{aligned}
|\tau(G \circ F)|(x) & =\sqrt{\sum_{\alpha}\left(\sum_{\beta, i} g_{\beta}^{\alpha} f_{i i}^{\beta}+\sum_{\beta, \gamma, i} g_{\beta \gamma}^{\alpha} f_{i}^{\beta} f_{i}^{\gamma}\right)^{2}} \\
& =\sqrt{\sum_{\alpha}\left(\sum_{\beta, \gamma, i} g_{\beta \gamma}^{\alpha} f_{i}^{\beta} f_{i}^{\gamma}\right)^{2}} \\
& \leq \epsilon \cdot e(F)(x) .
\end{aligned}
$$

Lemma 2.4. Let $G$ be a map from $\mathbb{H}^{m}$ to itself such that for some $\epsilon>0$ (and in some orthonormal coframes),

$$
\sqrt{\sum_{j, k}\left(\sum_{i} g_{i}^{j} g_{i}^{k}-\delta_{j k}\right)^{2}}<\epsilon .
$$


Then for any map $F: \mathbb{H}^{m} \rightarrow \mathbb{H}^{n}$, we have

$$
e(F \circ G)(x) \geq(1-\epsilon) e(F)(x), \quad \forall x \in \mathbb{H}^{m} .
$$

Proof. By a straight forward calculation,

$$
\begin{aligned}
e(F \circ G)(x) & =\sum_{\substack{1 \leq \alpha \leq n \\
1 \leq i \leq m}}\left(\sum_{j=1}^{m} f_{j}^{\alpha} g_{i}^{j}\right)^{2} \\
& =\sum_{\substack{1 \leq \alpha \leq n \\
1 \leq i, j, k \leq m}} f_{j}^{\alpha} f_{k}^{\alpha} g_{i}^{j} g_{i}^{k} \\
& =\sum_{\substack{1 \leq \alpha \leq n \\
1 \leq j, k \leq m}} f_{j}^{\alpha} f_{k}^{\alpha}\left(\sum_{i=1}^{m} g_{i}^{j} g_{i}^{k}-\delta_{j k}\right)+\sum_{\substack{1 \leq \alpha \leq n \\
1 \leq i \leq m}}\left(f_{i}^{\alpha}\right)^{2} \\
& \geq(-\epsilon+1) e(F)(x) .
\end{aligned}
$$

Similarly, the same calculation gives

Lemma 2.5. Let $G$ be a map from $\mathbb{H}^{n}$ to itself such that for some $\epsilon>0$ (and in some orthonormal coframes),

$$
\sqrt{\sum_{\beta, \gamma}\left(\sum_{\alpha} g_{\beta}^{\alpha} g_{\gamma}^{\alpha}-\delta_{\beta \gamma}\right)^{2}}<\epsilon .
$$

Then for any map $F: \mathbb{H}^{m} \rightarrow \mathbb{H}^{n}$, we have

$$
e(G \circ F)(x) \geq(1-\epsilon) e(F)(x), \quad \forall x \in \mathbb{H}^{m} .
$$

Now we are going to prove that if a quasi-conformal map on $\mathbb{S}^{n-1}$ has a quasi-conformal harmonic extension to $\mathbb{H}^{n}$, then 'nearby' quasiconformal maps on $\mathbb{S}^{n-1}$ also have harmonic extension which are pseudo-isometries.

Theorem 2.6. For any quasiconformal harmonic diffeomorphism $F$ on $\mathbb{H}^{n}$, there exists a $K_{0}>1$ such that if $G$ is a $K_{0}$-quasiconformal map on $\mathbb{S}^{n-1}$, then there is a pseudo-isometric harmonic map $H$ from $\mathbb{H}^{n}$ onto $\mathbb{H}^{n}$ such that $H=G \circ F$ on $\mathbb{S}^{n-1}$. If in addition, $F$ is a quasi-isometry, then there is also a pseudo-isometric harmonic map $\tilde{H}$ from $\mathbb{H}^{n}$ to $\mathbb{H}^{n}$ such that $\tilde{H}=F \circ G$ on $\mathbb{S}^{n-1}$.

Remark 2.7. It is easy to see that if we put $F$ equal to the identity map of $\mathbb{H}^{n}$, then we have the existence of pseudo-isometric harmonic extension to 
$\mathbb{H}^{n}$ for every quasiconformal map on $\mathbb{S}^{n-1}$ with small enough dilatation. In fact, we have a stronger result that the extension is quasi-isometric provided that the dilatation is small enough (perhaps smaller than that of the previous statement). We will prove this fact in Corollary 4.2.

Proof of Theorem 2.6. We use an argument as in [L-T 3]. By Proposition 1.2 , given $\epsilon>0$ and with respect to any orthonormal coframes, there is $K_{0}>1$ such that if $G$ is $K_{0}$-quasiconformal map on $\mathbb{S}^{n-1}$, then $G$ has a quasiconformal extension on $\mathbb{H}^{n}$, also denoted by $G$, such that $G$ is smooth in $\mathbb{H}^{n}$ and

$$
\left\{\begin{array}{l}
\sqrt{\sum_{\beta, \gamma}\left(\sum_{\alpha} g_{\beta}^{\alpha} g_{\gamma}^{\alpha}-\delta_{\beta \gamma}\right)^{2}}<\epsilon \\
\sqrt{\sum_{\beta, \gamma}\left(\sum_{\alpha} g_{\alpha}^{\beta} g_{\alpha}^{\gamma}-\delta_{\beta \gamma}\right)^{2}}<\epsilon
\end{array}\right.
$$

and

$$
\left|\nabla^{2} G\right| \leq \epsilon
$$

By Lemma 2.3, (2.2) and (2.3), we see that the norm of the tension field of $G \circ F$ satisfies

$$
|\tau(G \circ F)|(x) \leq \epsilon \cdot e(F)(x) .
$$

Fixing a point $o \in \mathbb{H}^{n}$, we can find, for any $R>0$, a unique harmonic map $H_{R}$ on $B_{o}(R)$ such that $H_{R}=G \circ F$ on $\partial B_{o}(R)$. We want to estimate $d_{R}(x)=d\left(H_{R}(x), G \circ F(x)\right)$ for $x \in B_{o}(R)$. In order to do so, we first choose, for $x \in \mathbb{H}^{n}$, an orthonormal frame $\left\{e_{1}, \ldots, e_{n}\right\}$ near $x$. Then consider the minimal geodesic $\gamma(t)$ from $H_{R}(x)$ to $G \circ F(x)$ parametrized by arclength and choose orthonormal frames $\left\{f_{1}, \ldots, f_{n}\right\}$ and $\left\{\bar{f}_{1}, \ldots, \bar{f}_{n}\right\}$ near $H_{R}(x)$ and $G \circ F(x)$ respectively such that $f_{n}=-\gamma^{\prime}(0)$ at $H_{R}(x)$ and $\bar{f}_{n}=\gamma^{\prime}(l)$ at $G \circ F(x)$. We may assume that all orthonormal frames are positively oriented. With these orthonormal frames, we write

$$
\left(H_{R}\right)_{*}\left(e_{i}\right)=\sum_{\alpha=1}^{n} u_{i}^{\alpha} f_{\alpha}
$$

and

$$
(G \circ F)_{*}\left(e_{i}\right)=\sum_{\alpha=1}^{n} v_{i}^{\alpha} \bar{f}_{\alpha},
$$

for $i=1, \ldots, n$. Then as in $[\mathbf{L}-\mathbf{T} \mathbf{3}]$, by results of $[\mathbf{S}-\mathbf{Y}, \mathbf{J}-\mathbf{K}]$, we have

$$
\Delta d_{R}(x) \geq-|\tau(G \circ F)|(x)+\sum_{\substack{1 \leq i \leq n \\ 1 \leq \alpha \leq n-1}}\left(\left(u_{i}^{\alpha}\right)^{2}(x)+\left(v_{i}^{\alpha}\right)^{2}(x)\right) \tanh \frac{d_{R}(x)}{2}
$$


in distributional sense.

Since $F$ and $G$ are quasi-conformal, so is $G \circ F$. The quasiconformal constants of all those $G$ are uniformly bounded by some constant provided that we are willing to restrict $\epsilon$ small enough, say $0<\epsilon<1 / 2$. Hence there is a constant $K_{1}$ depending only on the quasiconformal constant of $F$ such that

$$
e(G \circ F)(x) \leq K_{1} J^{\frac{2}{n}}(x),
$$

where $J=\operatorname{det}\left(v_{i}^{\alpha}\right)$ is the Jacobian of the map $G \circ F$. Let $V_{i}^{\alpha}$ be the cofactor of $v_{i}^{\alpha}$. For any $\eta>0$, we have

$$
\begin{aligned}
e(G \circ F) & \leq K_{1} J^{\frac{2}{n}} \\
& \leq K_{1}\left|v_{1}^{n} V_{1}^{n}+\cdots+v_{n}^{n} V_{n}^{n}\right|^{\frac{2}{n}} \\
& \leq C K_{1}\left(\left|v_{1}^{n} V_{1}^{n}\right|^{\frac{2}{n}}+\cdots+\left|v_{n}^{n} V_{n}^{n}\right|^{\frac{2}{n}}\right) \\
& \leq C K_{1}\left(\eta \sum_{i=1}^{n}\left(v_{i}^{n}\right)^{2}+\eta^{-\frac{1}{n-1}} \sum_{i=1}^{n}\left|V_{i}^{n}\right|^{\frac{2}{n-1}}\right)
\end{aligned}
$$

where $C$ is a constant depending only on $n$. Take $\eta>0$ so that $\eta C K_{1} \leq \frac{1}{2}$, we have

$$
e(G \circ F)(x) \leq C_{2} \sum_{\substack{1 \leq i \leq n \\ 1 \leq \alpha \leq n-1}}\left(v_{i}^{\alpha}\right)^{2}(x),
$$

for some constant $C_{2}$ depending only on the quasiconformal constant of $F$ and $n$. By (2.5), Lemmas 2.3 and 2.4, we have

$$
\begin{aligned}
\Delta d_{R}(x) & \geq-|\tau(G \circ F)|(x)+\frac{1-\epsilon}{C_{2}} e(F)(x) \cdot \tanh \frac{d_{R}(x)}{2} \\
& \geq e(F)(x)\left(-\epsilon+\frac{1-\epsilon}{C_{2}} \cdot \tanh \frac{d_{R}(x)}{2}\right) .
\end{aligned}
$$

Hence for $0<\epsilon<1 / 2$, by noting that $e(F)>0$, (2.6) and the maximum principle imply that

$$
d_{R}(x) \leq C_{3} \epsilon,
$$

where $C_{3}=2 C_{2}$ depends only on $n$ and the quasiconformal constant of $F$. It is then easy to see that, by passing to a subsequence, $H_{R}$ converges uniformly on compact sets to a harmonic map $H$ on $\mathbb{H}^{n}$ such that

$$
d(H(x), G \circ F(x)) \leq C_{3} \epsilon .
$$

Since $F$ is quasiconformal, $F$ is a rough isometry by $[\mathbf{P}]$. Hence $H$ is also a rough isometry by (2.7). Finally, by the estimate on the energy density 
of harmonic maps in [C], the energy density of $H$ is uniformly bounded. Therefore, $H$ is a pseudo-isometry, which proves the first part of the theorem.

If in addition $F$ is a quasi-isometry, we use Lemma 2.2 to estimate the tension field of $F \circ G$. Proceed as before, instead of (2.6), we have

$$
\begin{aligned}
\Delta d_{R}(x) & \geq-|\tau(F \circ G)|(x)+\frac{1-\epsilon}{C_{2}} e(F)(x) \cdot \tanh \frac{d_{R}(x)}{2} \\
& \geq-C_{1} \epsilon+\frac{1-\epsilon}{C_{2}} e(F)(x) \cdot \tanh \frac{d_{R}(x)}{2},
\end{aligned}
$$

where $C_{1}$ is a constant depending only on $n$ and the upper bound of the energy density of $F$ as in Lemma 2.2. Since $F$ is a quasi-isometry, $e(F)$ is bounded below by a positive constant. Hence we still have a harmonic map $H$ satisfying (2.7) with a new constant $C_{3}$ which depends only on $n$, the upper bound and the positive lower bound of the energy density of $e(F)$. This completes the proof of the theorem.

Remark 2.8. By (2.7), we see that for fixed $F, d(H(x), G \circ F(x)) \rightarrow 0$, and $d(\tilde{H}(x), G \circ F(x)) \rightarrow 0$ uniformly, as $\epsilon \rightarrow 0$. It is unclear whether $H$ and $\tilde{H}$ are quasiconformal or not. We will discuss this problem in $\S 4$.

\section{Construction of pseudo-isometric harmonic maps (II).}

We will consider a situation different from that in $\S 2$. It was proved in $\left[\mathbf{L}-\mathbf{T}\right.$ 1]-[L-T 3] that a smooth map from $\mathbb{S}^{m-1}$ to $\mathbb{S}^{n-1}$ with nowhere vanishing energy density can be extended to a proper harmonic map from $\mathbb{H}^{m}$ to $\mathbb{H}^{n}$. Moreover, the harmonic maps are $C^{m, \alpha}$ up to the boundary for some $0<\alpha<1$. Suppose $m=n$ and the boundary data is a diffeomorphism, then in view of Theorem 2.6, it is natural to ask whether the harmonic map constructed is a quasiconformal diffeomorphism, or even just a diffeomorphism. Unless $m=n=2$, the answer is unclear. Hence Theorem 2.6 cannot be applied to this class of maps. However, from its proof, it is easy to see that given a harmonic map $F$, one can construct 'nearby' harmonic map if $F$ behaves well near infinity. Hence we have a result similar to Theorem 2.6 for this case. Before giving the precise statement, we first introduce the following:

Definition 3.1. Let $F$ be a map from a complete noncompact manifold $M$ to itself. $F$ is said to be a quasi-isometry near infinity if there is a compact subset $B \subset \subset M$ and constant $C>1$ such that

$$
C d(x, \tilde{x}) \geq d(F(x), F(\tilde{x})) \geq C^{-1} d(x, \tilde{x}), \quad \forall x, \tilde{x} \in M \backslash B .
$$


Note that if $F$ is a quasi-isometry near infinity which is also continuous, then it must be a rough isometry.

Proposition 3.1. $\quad$ Let $F$ be a proper harmonic map from $\mathbb{H}^{n}$ to itself which is $C^{1}$ up to the boundary when considered as a map from the close unit ball in $\mathbb{R}^{n}$ to itself. Suppose also that the boundary map of $F$ is a $C^{1}$ diffeomorphism on $\mathbb{S}^{n-1}$. Then $F$ is a quasi-isometry near infinity. In particular, it is a rough isometry.

Proof. Let us first show that $F$ is a diffeomorphism locally near a boundary point. For this purpose, it is simpler to do the calculation in the upper half space model of $\mathbb{H}^{n}$. We identify both the domain and target with the upper half space of $\mathbb{R}^{n}$ endowed with the Poincaré metrics. If we use $\left(x_{1}, \ldots, x_{n}\right)$ and $\left(y_{1}, \ldots, y_{n}\right)$ to denote the coordinates on the domain and target respectively, then the metrics are given by

$$
d s_{\mathbb{H}^{n}}^{2}=\frac{\left(d x^{1}\right)^{2}+\cdots+\left(d x^{n}\right)^{2}}{\left(x^{n}\right)^{2}} \quad \text { and } \quad d s_{\mathbb{H}^{n}}^{2}=\frac{\left(d y^{1}\right)^{2}+\cdots+\left(d y^{n}\right)^{2}}{\left(y^{n}\right)^{2}}
$$

respectively. Then, the components of $d F$ is given by

$$
f_{j}^{i}=\frac{x^{n}}{y^{n}} \frac{\partial y^{i}}{\partial x^{j}}
$$

with respect to the orthonormal coframes $d x^{i} / x^{n}$ and $d y^{j} / y^{n}$. The results of [L-T 2] imply that

$$
\lim _{x^{n} \rightarrow 0} \frac{y^{n}}{x^{n}}=\sqrt{\frac{e(\phi)}{n-1}}>0,
$$

where $\phi$ is the boundary map, and hence

$$
\frac{\partial y^{n}}{\partial x^{n}}=\sqrt{\frac{e(\phi)}{n-1}}>0 .
$$

We also have

$$
\frac{\partial y^{i}}{\partial x^{n}}=0, \quad \text { at } x^{n}=0, \quad i=1, \ldots, n-1 .
$$

These imply that the Jacobian of $F$ approaches a positive multiple of the Jacobian of the boundary map $\phi$ as $x$ tends to the boundary. Since $\phi$ is a diffeomorphism, $F$ is diffeomorphism near a boundary point.

To show that $F$ is a diffeomorphism onto its image in a neighborhood of the whole boundary, we use the unit ball model of $\mathbb{H}^{n}$. Assume on the contrary 
that it is not true. Then, there exist two sequences $\left\{x^{k}\right\}$ and $\left\{\tilde{x}^{k}\right\}$ such that $x^{k} \neq \tilde{x}^{k}, \forall k, x^{k} \rightarrow \theta^{1} \in \mathbb{S}^{n-1}, \tilde{x}^{k} \rightarrow \theta^{2} \in \mathbb{S}^{n-1}$, and $F\left(x^{k}\right)=F\left(\tilde{x}^{k}\right)$ for all $k$. Since $F$ is a $C^{1}$ near $\theta^{1}$ and $\theta^{2}$, we have $\phi\left(\theta^{1}\right)=\phi\left(\theta^{2}\right)$, which implies that $\theta^{1}=\theta^{2}$. Hence, by the fact that $F$ is a diffeomorphism near a boundary point again, we see that $x^{k}=\tilde{x}^{k}$ for $k$ large, which is a contradiction. Hence we have proved that $F$ is a diffeomorphism near the whole boundary. More precisely, for any point $o \in \mathbb{H}^{n}$, there is $R_{0}>0$ such that $F$ is a diffeomorphism from $\mathbb{H}^{n} \backslash B_{o}\left(R_{0}\right)$ onto its image.

Since $F$ has bounded energy density by construction in [L-T 1]-[L-T 3] and the Jacobian of $F$ has a uniform positive lower bound outside $B_{o}\left(R_{0}\right)$ by the above arguments, the energy density of $F^{-1}$ is uniformly bounded on $F\left(\mathbb{H}^{n} \backslash B_{o}\left(R_{0}\right)\right)$. Hence there is a positive constant $a>0$, such that for any $x, y$ outside $B_{o}\left(R_{0}\right)$,

$$
d(F(x), F(y)) \geq \operatorname{ad}(x, y)
$$

provided that the minimal geodesic joining $F(x)$ and $F(y)$ lies inside $F\left(\mathbb{H}^{n} \backslash\right.$ $\left.B_{o}\left(R_{0}\right)\right)$.

In general, if $x, y \in \mathbb{H}^{n} \backslash B_{o}\left(R_{0}\right)$. Let $\gamma(t), 0 \leq t \leq L$ be the minimal geodesic joining $\tilde{x}=F(x)$ and $\tilde{y}=F(y)$. Let $t_{1}$ be the first $t$ such that $\gamma(t) \in \overline{F\left(B_{o}\left(R_{0}\right)\right)}$ and $t_{2}$ be the last $t$ such that $\gamma(t) \in \overline{F\left(B_{o}\left(R_{0}\right)\right)}$. And let $x_{1}$ and $y_{1}$ be the preimage of $\gamma\left(t_{1}\right)$ and $\gamma\left(t_{2}\right)$ respectively, i.e. $F\left(x_{1}\right)=\gamma\left(t_{1}\right)$ and $F\left(y_{1}\right)=\gamma\left(t_{2}\right)$ with $x_{1}, y_{1} \in \partial \overline{B_{o}\left(R_{0}\right)}$. Then, by (3.1), we have

$$
d\left(F(x), F\left(x_{1}\right)\right) \geq \operatorname{ad}\left(x, x_{1}\right) \quad \text { and } \quad d\left(F(y), F\left(y_{1}\right)\right) \geq a d\left(y, y_{1}\right) .
$$

Therefore

$$
\begin{aligned}
d(F(x), F(y)) & \geq a\left(d\left(x, x_{1}\right)+d\left(y, y_{1}\right)\right) \\
& \geq a d(x, y)-2 a R_{0}
\end{aligned}
$$

Finally, to complete our proof, we assume on the contrary that $F$ is not quasi-isometric near infinity. Then, there is a sequence of numbers $\epsilon_{i} \rightarrow 0$ and sequences of points $\left\{x_{i}\right\}$ and $\left\{y_{i}\right\} \rightarrow \infty$ such that

$$
d\left(F\left(x_{i}\right), F\left(y_{i}\right)\right)<\epsilon_{i} d\left(x_{i}, y_{i}\right)
$$

Then by (3.1) and (3.2), we have

$$
a d\left(x_{i}, y_{i}\right)-2 a R_{0}<\epsilon_{i} d\left(x_{i}, y_{i}\right)
$$

Since $\epsilon_{i} \rightarrow 0, d\left(x_{i}, y_{i}\right) \leq 3 R_{0}$ for $i$ large enough, and since $F$ has bounded energy density, $d\left(F\left(x_{i}\right), F\left(y_{i}\right)\right) \leq C$ for some constant $C$ provided $i$ large 
enough. Note that if $o^{\prime}$ is a fixed point in $\mathbb{H}^{n}$, then there is $R_{1}>0$ such that $\mathbb{H}^{n} \backslash B_{o^{\prime}}\left(R_{1}\right)$ is in the image of $\mathbb{H}^{n} \backslash B_{o}\left(R_{0}\right)$. Since $F$ is a proper map, and since $d\left(F\left(x_{i}\right), F\left(y_{i}\right)\right) \leq C$, for $i$ large, the minimal geodesic joining $F\left(x_{i}\right)$ and $F\left(y_{i}\right)$ is contained in $F\left(\mathbb{H}^{n} \backslash B_{o}\left(R_{0}\right)\right)$. So we can apply (3.1) and get

$$
a d\left(x_{i}, y_{i}\right)<\epsilon_{i} d\left(x_{i}, y_{i}\right)
$$

which is a contradiction.

Theorem 3.2. Let $F$ be a proper harmonic map from $\mathbb{H}^{m}$ to $\mathbb{H}^{n}$ which is $C^{1}$ up to the boundary when considered as a map from the close unit ball in $\mathbb{R}^{m}$ into the close unit ball of $\mathbb{R}^{n}$. Suppose also that the boundary map of $F$ is a smooth map from $\mathbb{S}^{m-1}$ to $\mathbb{S}^{n-1}$ with nowhere vanishing energy density. There exists an $K_{0}>1$, which is independent of $F$, such that if $G$ is a $K_{0}$-quasiconformal map on $\mathbb{S}^{n-1}$ (respectively $\mathbb{S}^{m-1}$ ), then there is a harmonic map $H$ from $\mathbb{H}^{m}$ into $\mathbb{H}^{n}$ such that $H=G \circ F$ (respectively $H=F \circ G)$ on $\mathbb{S}^{m-1}$ and that $\sup _{x \in M} d(H(x), G \circ F(x))<\infty$ (respectively $\left.\sup _{x \in M} d(H(x), F \circ G(x))<\infty\right)$. Here $G$ also denotes the Douady-Earle extension of $G$. If in addition, $m=n$ and the boundary map of $F$ is a diffeomorphism, then $H$ is a pseudo-isometry.

Proof. The proof is similar to that of Theorem 2.6. However we need more work especially in showing that $K_{0}$ is independent of $F$. Let us first consider $G \circ F$. As in the proof of Theorem 2.6, for any $R>0$, we can find uniquely a harmonic map $H_{R}$ on $B_{o}(R)$ with boundary value $G \circ F$. We have the same Bochner type formula for $d_{R}(x)=d_{\mathbb{H}^{m}}\left(H_{R}(x), G \circ F(x)\right)$. That is, for $x \in B_{o}(R)$, we have (using the same notations as in Theorem 2.6)

$$
\Delta d_{R}(x) \geq-|\tau(G \circ F)|(x)+\sum_{\substack{1 \leq i \leq m \\ 1 \leq \alpha \leq n-1}}\left(\left(u_{i}^{\alpha}\right)^{2}(x)+\left(v_{i}^{\alpha}\right)^{2}(x)\right) \cdot \tanh \frac{d_{R}(x)}{2}
$$

And as before, we want to estimate $\sum_{i=1}^{m} \sum_{\alpha=1}^{n-1}\left(v_{i}^{\alpha}\right)^{2}$ from below near the boundary. We will work locally at the origin of the upper half space model of both the domain and target as in the proof of Proposition 3.1. Note that the dimensions of the domain and target are $m$ and $n$ respectively which are not assumed to be equal. Hence the orthonormal coframes which we choose are now $d x^{i} / x^{m}$ and $d y^{\alpha} / y^{n}$. Therefore, the components of the differential of $F$ is given by $f_{i}^{\alpha}=\frac{x^{m}}{y^{n}} \frac{\partial y^{\alpha}}{\partial x^{i}}$. In these notations, since $F$ is $C^{1}$ up to boundary, the results of $[\mathbf{L}-\mathbf{T} \mathbf{2}],[\mathbf{L}-\mathbf{T} \mathbf{3}]$ imply that near the origin

$$
\begin{gathered}
\left|f_{m}^{\alpha}\right|+\left|f_{i}^{n}\right| \leq C_{4} x^{m}, \quad \text { for } 1 \leq \alpha \leq n-1 \text { and } 1 \leq i \leq m-1, \\
\left|f_{m}^{n}-1\right| \leq C_{4} x^{m},
\end{gathered}
$$


and

$$
\left|\sum_{\substack{1 \leq j \leq m-1 \\ 1 \leq \beta \leq n-1}}\left(f_{j}^{\beta}\right)^{2}-(m-1)\right| \leq C_{4} x^{m},
$$

for some constant $C_{4}$ depending only for $F$. Then

$$
\begin{aligned}
\sum_{\substack{1 \leq i \leq m \\
1 \leq \alpha \leq n-1}}\left(v_{i}^{\alpha}\right)^{2} & =\sum_{\substack{1 \leq i \leq m \\
1 \leq \alpha \leq n-1}}\left(\sum_{1 \leq \beta \leq n} g_{\beta}^{\alpha} f_{i}^{\beta}\right)^{2} \\
& =e(G \circ F)-\sum_{i=1}^{m}\left(\sum_{\beta=1}^{n} g_{\beta}^{n} f_{i}^{\beta}\right)^{2} \\
& =e(G \circ F)-\sum_{i=1}^{m-1}\left(\sum_{\beta=1}^{n} g_{\beta}^{n} f_{i}^{\beta}\right)^{2}-\left(\sum_{\beta=1}^{n} g_{\beta}^{n} f_{m}^{\beta}\right)^{2} .
\end{aligned}
$$

Here $\left(g_{\beta}^{\alpha}\right)$ is the matrix of $d G$ with respect to the corresponding orthonormal coframes. Then given any $1>\epsilon>0$, by Proposition 1.2, Lemma 2.3, and 2.5 , there is a $K_{0}>1$ such that for any $K_{0}$-quasiconformal map $G$ on $\mathbb{S}^{n-1}$, we have

$$
\begin{aligned}
|\tau(G \circ F)|(x) & \leq \epsilon e(F)(x), \\
e(G \circ F)(x) & \geq(1-\epsilon) e(F)(x),
\end{aligned}
$$

and

$$
\sqrt{\sum_{\beta, \gamma}\left(\sum_{\alpha=1}^{n} g_{\beta}^{\alpha} g_{\gamma}^{\alpha}-\delta_{\beta \gamma}\right)^{2}} \leq \epsilon .
$$

Recall that in these inequalities, $G$ denotes the Douady-Earle extension of the boundary homeomorphism. Hence by (3.4) and (3.6), if $x$ is near the origin in the upper space model for $\mathbb{H}^{m}$, with $x^{m}<1$,

$$
\begin{aligned}
\sum_{i=1}^{m-1}\left(\sum_{\beta=1}^{n} g_{\beta}^{n} f_{i}^{\beta}\right)^{2} & \leq \sum_{i=1}^{m-1}\left(\sum_{\beta=1}^{n-1}\left|g_{\beta}^{n} f_{i}^{\beta}\right|+C_{5} x^{m}\right)^{2} \\
& \leq\left(\sum_{i=1}^{m-1} \sum_{\beta=1}^{n}\left(f_{i}^{\beta}\right)^{2}\right)\left(\sum_{\beta=1}^{n-1}\left(g_{\beta}^{n}\right)^{2}\right)+C_{6} x^{m} \\
& \leq(m-1) \sum_{\beta=1}^{n}\left(g_{\beta}^{n}\right)^{2}-(m-1)\left(g_{n}^{n}\right)^{2}+C_{7} x^{m}
\end{aligned}
$$


where $C_{5}, C_{6}, C_{7}$ are constants depending only on $m, n$, and the upper bound of $e(F)$. By (3.4) and (3.5), we have, for $x^{m}<1$

$$
\begin{aligned}
\left(\sum_{\beta=1}^{n} g_{\beta}^{n} f_{m}^{\beta}\right)^{2} & \leq\left(\sum_{\beta=1}^{n-1}\left|g_{\beta}^{n} f_{m}^{\beta}\right|+\left|g_{n}^{n} f_{m}^{n}\right|\right)^{2} \\
& \leq\left(g_{n}^{n}\right)^{2}+C_{8} x^{m}
\end{aligned}
$$

where $C_{8}$ is a constant depending only on $m, n$ and the upper bound of $e(F)$. From (3.4)-(3.6), it is easy to see that $|e(F)-m| \leq C x^{m}$ near the origin for some constant depending on $m, n$ and $F$. Hence together with (3.9), (3.11) and (3.12), we have at $x$,

$$
\begin{aligned}
\sum_{\substack{1 \leq i \leq m \\
1 \leq \alpha \leq n-1}}\left(v_{i}^{\alpha}\right)^{2} & \geq e(G \circ F)-(m-1) \sum_{\beta=1}^{n}\left(g_{\beta}^{n}\right)^{2}+(m-1)\left(g_{n}^{n}\right)^{2}-\left(g_{n}^{n}\right)^{2}-C_{9} x^{m} \\
& \geq(1-\epsilon) e(F)-(m-1) \sum_{\beta=1}^{n}\left(g_{\beta}^{n}\right)^{2}-C_{9} x^{m} \\
& \geq m-m \epsilon-(m-1)(1+\epsilon)-C_{10} x^{m} \\
& \geq 1-C_{10} x^{m}-(2 m-1) \epsilon
\end{aligned}
$$

where $C_{9}, C_{10}$ are constants depending only on $m, n$, and $F$. Combining this with (3.3) and (3.8), we have the following: For any given $\eta>0$, there is $R_{0}>0$ depending only on $\eta$ and $F$ such that if $R>R_{0}$ and $x \in B_{o}(R) \backslash B_{o}\left(R_{0}\right)$, then

$$
\Delta d_{R}(x) \geq-m \epsilon-C_{11} \eta+\left(1-C_{10} \eta-(2 m-1) \epsilon\right) \cdot \tanh \frac{d_{R}}{2},
$$

where $C_{11}$ depends on $m, n$ and $F$. Note that (3.3) and (3.8) also imply that for $x$ inside $B_{o}\left(R_{0}\right)$,

$$
\Delta d_{R}(x) \geq-C_{12} \epsilon
$$

where $C_{12}$ is a constant depending only on $m, n$, and the upper bound of $e(F)$. It is easy to see that there are constants $a, b>0$ such that

$$
\Delta \exp \left(-a \sqrt{r^{2}+1}\right) \leq-b \exp \left(-a \sqrt{r^{2}+1}\right) \text { on } \mathbb{H}^{m},
$$

where $r(x)=d(o, x)$. If we take $C_{13}=\frac{2 C_{12}}{b \exp \left(-a \sqrt{R_{0}^{2}+1}\right)}$ depending only on $m$, $n, \eta$, and $F$. Then

$$
\Delta\left\{C_{13} \epsilon \exp \left(-a \sqrt{r^{2}+1}\right)\right\} \leq-2 C_{12} \epsilon,
$$


on $B_{o}\left(R_{0}\right)$. If we first choose $\epsilon$ small so that $m \epsilon /(1-(2 m-1) \epsilon)<1 / 2$, i.e. $\epsilon<1 /(4 m-1)$, and then choose $\eta>0$ small so that $1-C_{10} \eta-(2 m-1) \epsilon>0$ and $\left(m \epsilon+C_{11} \eta\right) /\left(1-C_{10} \eta-(2 m-1) \epsilon\right)<1 / 2$, then the equation

$$
\tanh \frac{z}{2}=\frac{m \epsilon+C_{11} \eta}{1-C_{10} \eta-(2 m-1) \epsilon}
$$

has a unique positive solution

$$
z=d(\epsilon, \eta)=\tanh ^{-1}\left(\frac{m \epsilon+C_{11} \eta}{1-C_{10} \eta-(2 m-1) \epsilon}\right) .
$$

From (3.13), (3.14), and (3.15), we conclude that

$$
d_{R}(x)-C_{13} \epsilon \exp \left(-a \sqrt{r^{2}(x)+1}\right) \leq d(\epsilon, \eta) .
$$

Hence $d_{R}$ are uniformly bounded. By passing to a subsequence, $H_{R}$ converges uniformly on compact subset to a harmonic map $H$ with same boundary data at infinity as $G \circ F$ such that

$$
d(H(x), G \circ F(x)) \leq C_{13} \epsilon \exp \left(-a \sqrt{r^{2}(x)+1}\right)+d(\epsilon, \eta) .
$$

Since the smallness requirement for $\epsilon$ so that (3.17) holds does not depend on $F$, the $K_{0}$ we obtained is also independent of $F$. This proves the first part of the theorem for the case $G \circ F$.

If in addition, $m=n$ and the boundary map of $F$ is a diffeomorphism, then $F$ is a pseudo-isometry by Proposition 3.1. Hence using the result of $[\mathbf{C}]$ and (3.17), $H$ is also a pseudo-isometry.

The case for $F \circ G$ is similar, we leave the details of the proof to the readers.

Remark 3.3. Note that from (3.17) and that $C_{13}$ depends only on $m, n$, $\eta$, and $F$, it is easy to see that

$$
\limsup _{x \rightarrow \infty} d(H(x), G \circ F(x)) \leq d(\epsilon, \eta)
$$

uniformly in $G$ and $H$. Note also that $d(\epsilon, \eta) \rightarrow 0$ as $\epsilon$ and $\eta$ both tend to 0 . We will use these facts in $\S 4$.

\section{Quasi-isometric harmonic maps.}

By [Sa, S-Y, C], if $n=2$, then the harmonic maps constructed in Theorem 2.6 are harmonic diffeomorphism with bounded energy density, see also 
[T-W]. In fact, the harmonic maps are quasi-isometries by the result of $[\mathbf{W}$. In particular, they are quasiconformal. On the other hand, it was proved in $[\mathbf{W}]$ that any quasiconformal harmonic map from $\mathbb{H}^{2}$ onto $\mathbb{H}^{2}$ is a quasi-isometry. However, it is unclear whether these results are still true in higher dimensions. In this section we will discuss this kind of problems. The first result in this section is to show that Theorem 2.6 can be sharpened in some cases. More precisely, we have:

Theorem 4.1. Let $F$ be a quasi-isometric harmonic diffeormorphism on $\mathbb{H}^{n}$. Then there exists $K_{0}>1$ such that if $G$ is a $K_{0}$-quasiconformal map on $\mathbb{S}^{n-1}$, then there are quasi-isometric harmonic maps $H$ and $\tilde{H}$ from $\mathbb{H}^{n}$ onto $\mathbb{H}^{n}$ such that $H=G \circ F$ and $\tilde{H}=F \circ G$ on $\mathbb{S}^{n-1}$.

Proof. We will prove the case for $G \circ F$, the other case is similar. Suppose the theorem is not true for this case, then there exists a sequence $K_{i}>1, K_{i} \rightarrow 1$, and $K_{i}$-quasiconformal map $G_{i}$ on $\mathbb{S}^{n-1}$, such that each of the harmonic map $H_{i}$ constructed in Theorem 2.6 with the same boundary value as $G_{i} \circ F$ is not a quasi-isometry for all $i$. We again denote the Douady-Earle extension of $G_{i}$ by the same symbol $G_{i}$ as before. By the construction in Theorem 2.6, Remark 2.8 and the fact that $F$ is a quasi-isometry, we can find positive constants $a$ and $b$ such that

$$
a^{-1} d(x, \tilde{x})-b \leq d\left(H_{i}(x), H_{i}(\tilde{x})\right) \leq a d(x, \tilde{x})
$$

for all $x, \tilde{x} \in \mathbb{H}^{n}$ and for all $i$. Since $H_{i}$ is not a quasi-isometry, there are $x_{i}, \tilde{x}_{i}$ such that

$$
d\left(H_{i}\left(x_{i}\right), H_{i}\left(\tilde{x}_{i}\right)\right)<\frac{1}{i} d\left(x_{i}, \tilde{x}_{i}\right) .
$$

From (4.1) and (4.2), we see that

$$
d\left(x_{i}, \tilde{x}_{i}\right) \leq 2 a b
$$

if $i$ is large enough.

Fix a point $o \in \mathbb{H}^{n}$ and let $\sigma_{i}$ and $\tau_{i}$ be the isometries of $\mathbb{H}^{n}$ such that $\tau_{i}(o)=x_{i}$ and $\sigma_{i} \circ H_{i}\left(x_{i}\right)=o$. Consider two sequences of maps $u_{i}=\sigma_{i} \circ H_{i} \circ \tau_{i}$ and $v_{i}=\sigma_{i} \circ\left(G_{i} \circ F\right) \circ \tau_{i}$. Since $H_{i}$ are harmonic and $\sigma_{i}$ and $\tau_{i}$ are isometries, we see that $u_{i}$ are harmonic maps such that $u_{i}(o)=o$ and satisfying

$$
a^{-1} d(x, \tilde{x})-b \leq d\left(u_{i}(x), u_{i}(\tilde{x})\right) \leq a d(x, \tilde{x}) .
$$

In particular, $\sup _{i, x} e\left(u_{i}\right)(x)<\infty$ by the gradient estimate of $[\mathbf{C}]$. Hence, a subsequence of $u_{i}$, which will also be denoted by $u_{i}$, converges uniformly on compact subsets of $\mathbb{H}^{n}$ to a harmonic map $u$. 
For the maps $v_{i}$, we observe, from Proposition 1.2, that there is a constant $\delta_{1}>0$ independent of $i$ such that, for all $i$ large enough,

$$
d\left(G_{i} \circ F(x), G_{i} \circ F(\tilde{x})\right) \geq \delta_{1} d(x, \tilde{x}),
$$

for all $x, \tilde{x} \in \mathbb{H}^{n}$. Therefore, from the definition of $v_{i}$, we also have

$$
d\left(v_{i}(x), v_{i}(\tilde{x})\right) \geq \delta_{1} d(x, \tilde{x}) .
$$

Now by the construction in Theorem 2.6, see Remark 2.8, we have

$$
\lim _{i \rightarrow \infty} \sup _{x \in \mathbb{H}^{n}} d\left(H_{i}(x), G_{i} \circ F(x)\right)=0,
$$

which implies that

$$
\lim _{i \rightarrow \infty} d\left(u_{i}(x), v_{i}(x)\right)=0, \quad \forall x \in \mathbb{H}^{n} .
$$

Combining this with (4.5), we have for all $x, \tilde{x} \in \mathbb{H}^{n}$,

$$
\begin{aligned}
d(u(x), u(\tilde{x})) & =\lim _{i \rightarrow \infty} d\left(u_{i}(x), u_{i}(\tilde{x})\right) \\
& \geq \lim _{i \rightarrow \infty}\left(d\left(v_{i}(x), v_{i}(\tilde{x})\right)-d\left(v_{i}(x), u_{i}(x)\right)-d\left(v_{i}(\tilde{x}), u_{i}(\tilde{x})\right)\right) \\
& \geq \delta_{1} d(x, \tilde{x}) .
\end{aligned}
$$

So $u$ is a quasi-isometry from (4.4) and (4.6). In particular, there is a positive constant $\delta_{2}$ such that

$$
J(u)(x) \geq \delta_{2}
$$

for all $x \in \mathbb{H}^{n}$. Since $u_{i}$ converges to $u$ in the $C^{\infty}$ norm uniformly on compact subsets of $\mathbb{H}^{n}$, for all $x \in B_{o}(4 a b)$ and $i$ large enough,

$$
J\left(u_{i}\right)(x) \geq \frac{1}{2} \delta_{2} .
$$

Note that the Hessian of $u_{i}$ are also uniformly bounded on compact sets, (4.7) implies that there is $r>0$ and positive constant $\delta_{3}$ such that for all $x \in B_{o}(r)$, wehave

$$
d\left(u_{i}(o), u_{i}(x)\right) \geq \delta_{3} d(o, x)
$$

for large $i$.

Now let us consider $y_{i}=\tau_{i}^{-1}\left(\tilde{x}_{i}\right)$. By (4.3) and the definition of $\tau_{i}$, we have $y_{i} \in B_{o}(2 a b)$. By (4.6) and the fact that $u_{i} \rightarrow u$ uniformly on compact subsets, if $d\left(o, y_{i}\right) \geq r$, then we have

$$
d\left(u_{i}(o), u_{i}\left(y_{i}\right)\right) \geq \frac{\delta_{1}}{2} r
$$


provided $i$ is large enough. Otherwise, (4.8) implies that

$$
d\left(u_{i}(o), u_{i}\left(y_{i}\right)\right) \geq \delta_{3} d\left(o, y_{i}\right) .
$$

In any case, we have contradiction since (4.2) and the definitions of $u_{i}$ together imply

$$
d\left(u_{i}(o), u_{i}\left(y_{i}\right)\right)<\frac{1}{i} d\left(o, y_{i}\right) .
$$

Hence the first part of the theorem is proved. The second part of the theorem can be proved similarly.

As we mentioned in Remark 2.7, if we take $F$ to be the identity map, we have:

Corollary 4.2. There exists $K_{0}>1$ depending only on $n$ such that if $G$ is a $K_{0}$-quasiconformal map on $\mathbb{S}^{n-1}$, then there is a harmonic quasi-isometry diffeomorphism $H$ on $\mathbb{H}^{n}$ such that $H=G$ on $\mathbb{S}^{n-1}$.

Using Theorem 3.2, and Remark 3.3, one can prove a result similar to Theorem 4.1. We leave the details of the proof to the readers.

Theorem 4.3. Let $F$ be a proper harmonic map from $\mathbb{H}^{n}$ to $\mathbb{H}^{n}$ which is $C^{1}$ up to the boundary when considered as a map from the close unit ball in $\mathbb{R}^{n}$ into the close unit ball of $\mathbb{R}^{n}$. Suppose also that the boundary map of $F$ is a diffeomorphism on $\mathbb{S}^{n-1}$. There exists $K_{0}>1$ such that if $G$ is a $K_{0}$-quasiconformal map on $\mathbb{S}^{n-1}$, then there are harmonic maps $H$ and $\tilde{H}$ from $\mathbb{H}^{n}$ into $\mathbb{H}^{n}$ such that $H=G \circ F$ and $\tilde{H}=F \circ G$. Moreover $H$ and $\tilde{H}$ are quasi-isometries near infinity.

As mentioned before, if $n \geq 3$, it is unknown whether a rough isometric harmonic map, or even a quasiconformal harmonic diffeomorphism, on $\mathbb{H}^{n}$ is a quasi-isometry. However, Theorem 4.1 and the uniqueness theorem in $[\mathbf{L}-\mathbf{W g}]$ on rough isometric harmonic maps give the following:

Corollary 4.4. There exists $K_{0}>1$ depending only on $n$ such that if $H$ is a rough isometric harmonic map on $\mathbb{H}^{n}$ with $K_{0}$-quasiconformal boundary data on $\mathbb{S}^{n-1}$, then $H$ is a quasi-isometry. More generally, if $F$ is a quasiisometric harmonic map on $\mathbb{H}^{n}$, then there is a $K_{0}>1$ such that if $H$ is a rough isometric harmonic map on $\mathbb{H}^{n}$ and $H \circ F^{-1}$ or $F^{-1} \circ H$ is a $K_{0}$ quasiconformal map when restricted on the geometric boundary of $\mathbb{H}^{n}$, then $H$ is a quasi-isometry.

The first part of Corollary 4.4 can be considered as a partial generalization of the result in $[\mathbf{W}]$ for dimension two that a quasiconformal harmonic map is 
a quasi-isometry. On the other hand, if we assume that $H$ is a quasiconformal map to begin with, then we can get a more explicit estimate of $K_{0}$.

Lemma 4.5. Let $F$ be a $K$-quasiconformal analytic map from $\mathbb{H}^{n}$ onto itself. Then all critical points (if any) of $F$ are isolated. Moreover, if o is a critical point of $F$, then

$$
e(F)(x)=O\left(d^{k}(x, o)\right), \quad \text { as } x \rightarrow o,
$$

for some integer $k$ satisfying

$$
2 \leq k \leq 2\left(K^{\frac{1}{n-1}}-1\right) .
$$

In particular, $F$ has no critical point if $K<2^{n-1}$.

Proof. It is an easy fact that critical points of analytic homeomorphisms are isolated. To prove the second part, we first observe that $e(F)(x)=$ $O\left(d^{k}(x, o)\right)$ for some $k$ since $F$ is analytic. To estimate $k$, we note that $e(F) \geq 0$ implies that if $e(F)(o)=0$, then $\nabla e(F)(o)=0$. Therefore, $k \geq 2$.

For the other side of the inequality, we may assume, by composing an isometry of $\mathbb{H}^{n}$ to $F$ from the left, that $F(o)=o$. Then since $F^{-1}$ is also a quasiconformal map from $\mathbb{H}^{n}$ onto itself with the same quasiconformal constant, we have by the Hölder estimate of quasiconformal maps by $[\mathbf{F}-\mathbf{V}]$ that

$$
d(x, o) \leq C_{1}(d(F(x), o))^{K^{1 /(1-n)}}
$$

for some constant $C_{1}$ and for all $x$ with $d(x, o) \leq 1$. Now, from $e(F)(x)=$ $O\left(d^{k}(x, o)\right)$, we conclude that for $d(x, o) \leq 1$,

$$
d(F(x), o) \leq C_{2} d^{1+\frac{k}{2}}(x, o),
$$

for some constant $C_{2}$. Combining this with (4.11), we have

$$
d(x, o) \leq C_{3}(d(x, o))^{\left(1+\frac{k}{2}\right) K^{1 /(1-n)}}, \quad \text { as } x \rightarrow o .
$$

Therefore, we must have

$$
\left(1+\frac{k}{2}\right) K^{1 /(1-n)} \leq 1
$$

which is the desired inequality.

Finally, the last statement is a direct consequence of above.

Applying the Lemma 4.5 to quasiconformal harmonic maps, we have 
Proposition 4.6. Let $F$ be a K-quasiconformal harmonic map on $\mathbb{H}^{n}$. Then all critical points (if any) of $F$ are isolated. Moreover, if o is a critical point of $F$, then

$$
e(F)(x)=O\left(d^{k}(x, o)\right), \quad \text { as } x \rightarrow o,
$$

for some integer $k$ satisfying

$$
2 \leq k \leq 2\left(K^{\frac{1}{n-1}}-1\right) .
$$

Suppose further that $K<2^{n-1}$, then $F$ is a quasi-isometry.

Proof. Only the last statement need a proof since all others follows easily from the analyticity of continuous harmonic maps and Lemma 4.5.

For the last statement, it is sufficient to prove that the energy density is uniformly bounded below away from 0 . In fact, if this is true, then the Jacobian of $F$ is also uniformly bounded below away from 0 , because $F$ is quasiconformal. On the other hand, we have uniformly upper bounded for the energy density of $F$ from the assumption that $F$ is quasiconformal. Hence $F$ is a pseudo-isometry. Again combining these two bounds, we see that the energy density of $F^{-1}$ is uniformly bounded from above and hence $F$ is a quasi-isometry.

We will prove that the energy density is uniformly bounded below away from 0 by contradiction. Suppose not, then there exists a sequence of points $x_{i} \in \mathbb{H}^{n}$, such that $e(F)\left(x_{i}\right) \rightarrow 0$ as $i \rightarrow \infty$. Let $o \in \mathbb{H}^{n}$ be a fixed point and $\sigma_{i}$ and $\tau_{i}$ be isometries of $\mathbb{H}^{n}$ such that $\tau_{i}(o)=x_{i}$ and $\sigma_{i}\left(F\left(x_{i}\right)\right)=o$. Then $u_{i}=\sigma_{i} \circ F \circ \tau_{i}$ are harmonic maps such that $u_{i}(o)=o$ and $e\left(u_{i}\right)(o) \rightarrow 0$ as $i \rightarrow \infty$. Moreover, each $u_{i}$ is a $K$-quasiconformal map. By $[\mathbf{F}-\mathbf{V}]$ for example, see also $[\mathbf{V}]$, a subsequence of $u_{i}$, also denoted by $u_{i}$, converges uniformly to a $K$-quasiconformal map $u$ on the close unit ball in $\mathbb{R}^{n}$, if we use the Poincaré disk model for $\mathbb{H}^{n}$. It is easy to see that $u$ is also harmonic with $u(o)=o$ and $e(u)(o)=0$. This obviously contradicts the last statement of Lemma 4.5. Hence the proof of the proposition is completed.

\section{References}

[B-A] A. Beurling and L.V. Ahlfors, The boundary correspondence under quasi-conformal mappings, Acta Math., 96 (1956), 125-142.

[C] S.Y. Cheng, Liouville theorem for harmonic maps, Proc. Symp. Pure Math., 36 (1980), 147-151.

[C-T] H.I. Choi and A. Treibergs, New examples of harmonic diffeomorphisms of hyperbolic plane, Manu. Math., 62 (1988), 249-256. 
[D-E] A. Douady and C.J. Earle, Conformally natural extension of homeomorphisms of the circle, Acta Math., 157 (1986), 23-48.

[F-V] R. Fehlmann and M. Vuorinen, Mori's theorem for n-dimensional quasiconformal mappings, Ann. Acad. Sci. Fenn. Ser. A I Math., 13 (1988).

[H-W] R. Hardt and M. Wolf, Harmonic extensions of quasiconformal maps to hyperbolic space, Indiana U. Math. J., 46 (1997), 155-163.

[J-K] W. Jäger and H. Kaul, Uniqueness of harmonic mappings and of solutions of elliptic equations on Riemannian manifolds, Math. Ann., 240 (1979), 231-250.

[L-T 1] P. Li and L.F. Tam, The heat equation and harmonic maps of complete manifolds, Invent. Math., 105 (1991), 1-46.

[L-T 2] _ Uniqueness and regularity of proper harmonic maps, Ann. Math., 136 (1992), 169-203.

[L-T 3] Uniqueness and regularity of proper harmonic maps II, Indiana U. Math. J., 42 (1993), 593-635.

[L-Wg] P. Li and J.-P. Wang, On harmonic rough-isometries, preprint.

[M] G.D. Mostow, Strong rigidity of locally symmetric spaces, Princeton University Press and Univeristy of Tokyo Press, 1973.

[P] P. Pansu, Quasiconformal mappings and manifolds of negative curvature, Lecture Notes in Mathematics, 1201 (1985), 212-229.

[Sa] J.H. Sampson, Some properties and applications of harmonic mappings, Ann. Sci. Ecole Normale Sup., 11 (1978), 211-228.

[S] R. Schoen, A role of harmonic mappings in rigidity and deformation problems, in collection: Complex Geometry (Osaka, 1990), Lecture Notes in Pure and Applied Mathematics, Dekker, New York, 143 (1993), 179-200.

[S-Y] R. Schoen and S.T. Yau, On univalent harmonic maps between surfaces, Invent. Math., 44 (1978), 265-278.

[T-W] L.-F. Tam and T. Wan, Quasi-conformal harmonic diffeomorphism and the universal Teichmüller space, J. Differential Geom., 42 (1995), 368-410.

[V] M. Vuorinen, Conformal Geometry and Quasiregular Mappings, Springer-Verlag Lecture Notes in Mathematics, 1319 (1988).

[W] T. Wan, Constant mean curvature surface, harmonic maps and universal Teichmüller, J. Diff. Geom., 35 (1991), 643-657.

[Wg] J.-P. Wang, Ph. D. Thesis, U. of California at Irvine, 1993.

[Y] D. Yang, Deforming a map into a harmonic map, preprint.

Received July 25, 1996. The authors are partially supported by Earmarked Grant, Hong Kong.

The Chinese University of Hong Kong

Shatin, N.T.

Hong Kong

E-mail address: lftam@math.cuhk.edu.hk

tomwan@math.cuhk.edu.hk

Note: The RefEREnCE ON P. 361 to Definition 3.1 APPEARED IN THE PAPER VERSion as Definition 2.1. Also, the equation (1.1) Was numbered (2.1) there. 